\title{
Qijiaoshengbai Capsule for Leukopenia: A Systematic Review and Meta-Analysis of Randomized Controlled Trials
}

\author{
Hongfang Fu $\mathbb{D}^{1,2}$, Yilan Wang $\mathbb{D}^{1,2}$ Haoyue Feng, ${ }^{1,2}$ Yang Zhang $\mathbb{D}^{1,2}$ and Xiaoyu Hu $\mathbb{D}^{1}$ \\ ${ }^{1}$ Hospital of Chengdu University of Traditional Chinese Medicine, Chengdu 610072, Sichuan Province, China \\ ${ }^{2}$ School of Clinical Medicine, Chengdu University of Traditional Chinese Medicine, Chengdu 611137, Sichuan Province, China \\ Correspondence should be addressed to Xiaoyu Hu; xiaoyuhu202010@163.com
}

Received 14 July 2021; Accepted 28 September 2021; Published 23 October 2021

Academic Editor: Oliver Micke

Copyright (C) 2021 Hongfang Fu et al. This is an open access article distributed under the Creative Commons Attribution License, which permits unrestricted use, distribution, and reproduction in any medium, provided the original work is properly cited.

Qijiaoshengbai capsule (QJSBC) is a type of proprietary Chinese medicine, which is an effective treatment for leukopenia in clinical practice. The purpose of this study is to evaluate the efficacy of QJSBC in improving specific clinical indicators, in patients with leukopenia of various origins. A total of seven electronic databases were searched, up until an end date of April 30, 2021, and a selection of clinical indicators was noted. The primary indicators of concern were related to blood: white blood cells (WBC). Secondary indicators were hemoglobin ( $\mathrm{Hb}$ ), platelets (PLT), neutrophils (NEU), bone marrow suppression rate (BMSR), and effective rate (ER). The methodological quality of the included trials was analyzed using a risk of bias assessment, as per the Cochrane Manual. The meta-analysis was performed using RevMan 5.4. Results. Twenty-four randomized controlled trials involving a total of 2,692 participants were included in this review. We found that QJSBC had a positive effect on increasing WBC, HB, PLT, and NEU and improving BMSR and ER. Conclusion. When compared with conventional chemotherapy (CC), conventional radiotherapy (CR), combined chemotherapy and radiotherapy $(\mathrm{CC}+\mathrm{CR})$, or conventional treatment $(\mathrm{CT})$, the use of QJSBC combined therapy can effectively improve the clinical outcome for patients with leukopenia. However, a larger sample size and a more standardized, high-quality study are required to validate these results.

\section{Introduction}

Leukopenia is a common blood disease with an incidence of $12.4 \%[1]$, in which the peripheral blood leukocyte count is less than $4 \times 10^{9} / \mathrm{L}$ [2]. Patients with mild symptoms may experience fatigue, dizziness, palpitations, loss of appetite, low fever, and chills. In addition, on top of this, patients with severe symptoms may also suffer from the common upper respiratory tract, urinary tract, and secondary infections, with their condition characterized by repeated attacks and difficult recovery $[3,4]$. The clinical etiology of leukopenia is complicated, with drug-induced leukopenia being the most common [5]. Examples include radiotherapy and chemotherapy drugs and antithyroid, psychiatric, and antihepatitis medications [6]. Leukopenia is seen in up to $70 \%$ of tumor patients after chemotherapy [7]. Severe bone marrow suppression (BMS) caused by chemotherapeutic drugs is the main reason that cancer patients are not able to continue chemotherapy, as it seriously affects the efficacy of treatment, prolongs the length of hospital stays, increases the cost to patients, and in some cases can even be fatal [8]. One study [9] showed that patients aged 41-70 years were most likely to be diagnosed with leukopenia. A possible reason for this may be that leukopenia is related to a change of pharmacokinetics in this age group; however, it should be noted that this is also consistent with the age distribution of cancer patients in China (showing a peak between 45 and 74 years of age). At present, granulocyte colony-stimulating factor (G-CSF) is commonly used in the treatment of leukopenia [10]. However, the high price of this drug creates a heavy economic burden on patients, and with common side effects such as skeletal muscle pain, low fever, and increased tumor risk, it is not optimal [11].

In China, traditional Chinese medicine (TCM) has shown good safety and efficacy in the prevention and treatment of leukopenia and BMS. Various prevention and 
treatment methods used include a medicated diet, acupuncture, moxibustion, and Chinese herbal medicine and they all have wide application and can be implemented in various forms [12-15]. Capsules of Qijiaoshengbai (QJSBC) is a proprietary Chinese medicine used in the treatment of leukopenia of various etiologies (Approval No. Z20025027). It is composed of seven authentic Guizhou herbs: Chinese jujube (da zao), blood ginseng (xue ren shen), Astragalus membranaceus (huang qi), Colla corii asini (e jiao), Angelica sinensis (dang gui), Sophora flavescens (ku shen), and Epimedium (yin yang huo) [16]. Since its launch, QJSBC has become one of the more commonly used Chinese patent medicines used in China to treat leukopenia. Studies have found that QJSBC has antitumor properties; it improves immunity, increases WBC and PLT counts, inhibits oxidation, and improves tissue perfusion [17].

In 2015, a systematic review and meta-analysis of QJSBC in the treatment of leukopenia was published in China [4]. However, that paper is now outdated as it does not include more recent literature published between 2015 and 2021, and there are other flaws. In this study, we evaluate the efficacy of QJSBC in preventing leukopenia and alleviating BMS via rigorous systematic evaluation and meta-analysis, to facilitate evidence-based medicine for clinical treatment.

\section{Methods}

2.1. Search Strategy. The systematic review and metaanalysis was based on the Preferred Reported Items for Systematic Reviews and Meta-Analysis (PRISMA) statement [18]. The following seven databases were searched from their inception up until April 30, 2021: PubMed, Embase, Cochrane Library, China National Knowledge Infrastructure (CNKI) database, Wanfang Data Knowledge Service Platform, the VIP information resource integration service platform (cqVIP), and China Biology Medicine Disc (Sino Med). The keywords used to search for randomized controlled trials (RCTs) were "qijiaoshengbai" OR "qi-jiao-sheng-bai" OR "qijiaoshengbaijiaonang" OR "qijiaoshengbai capsule," "leukopenia" OR "hypoleucocytosis" OR "hypolekocytosis" OR "marrow suppression" OR "bone marrow suppression" OR "bone marrow suppression rate," and "clinical trial" OR "randomized controlled trial." All the citations were searched and screened by two authors to reduce the risk of leaving out any relevant references. There was no limit imposed on the language of the search results.

\subsection{Selection Criteria}

\subsubsection{The Review Included RCTs That Met the Criteria Listed below}

(1) Patients had an established diagnosis of leukopenia;

(2) The treatment group was treated with QJSBC or QJSBC combined with other methods, while the control group was treated with non-QJSBC;

(3) Outcome indicators included WBC, PLT, Hb, NEU, BMSR (with evaluation criteria referring to the WTO
Anticancer Drug Classification Standard) [19] and ER.

There were no limitations in terms of gender, race, or country.

\subsubsection{Literature Was Excluded If It Met the following} Criteria. Duplicate studies, review articles, animal experiments, systematic evaluation, graduation papers, retrospective, cross-sectional studies, and conference abstracts.

2.3. Study Selection and Data Extraction. Two researchers selected the literature used in our study according to the inclusion and exclusion criteria after independently reading the title, abstract, and full text of each study. A data extraction template was created to extract the following information: first author, publication year, number of participants sampled, age, gender, intervention method, and course of treatment. Discrepancies between the two authors were resolved through discussion.

2.4. Quality Assessment and Statistical Analysis. The methodological quality of the selected trials was assessed according to the Cochrane Collaboration's tool [20]. Review Manager Version 5.4 was used to create a forest plot and conduct subgroup analysis. Relative risk (RR) was used for enumeration data; mean difference (MD) or standardized mean difference (SMD) was used for measurement data; and the range was expressed as the $95 \%$ confidence intervals (CI). Statistical heterogeneity was considered significant when $p<0.1$ and $I^{2}>50 \%$, and in this case, a random effects model was used to calculate the effect size. When $p<0.1$ and $I^{2}<50 \%$, the studies included were considered homogeneous and a fixed effects model was applied. Sensitivity analysis was conducted to test whether the result was robust by excluding the studies individually and comparing the effects of the remaining studies with the total effects of all the studies. Publication bias was assessed using a funnel plot analysis.

\section{Results}

3.1. Study Selection. From a total of 200 potentially related studies found during our initial search, 98 records were evaluated after the exclusion of 102 duplicates. Based on the titles and abstracts, an additional 52 studies were excluded as they were either a review, systematic evaluation, animal experiment, graduation paper, or conference abstract. This left 46 full-text articles, from which we excluded 22 trials because they were not randomized, the data were not reliable, or the outcomes measured did not meet our requirements. Therefore, a final total of 24 studies [21-44], including 1,424 patients in the experimental group and 1,268 patients in the control group, were selected for this study. A schematic of the selection process is shown in Figure 1.

3.2. Study Characteristics. Table 1 presents the main characteristics of the included trials. In total, 24 studies were selected, with 2,692 participants ranging in age from 14 to 81 

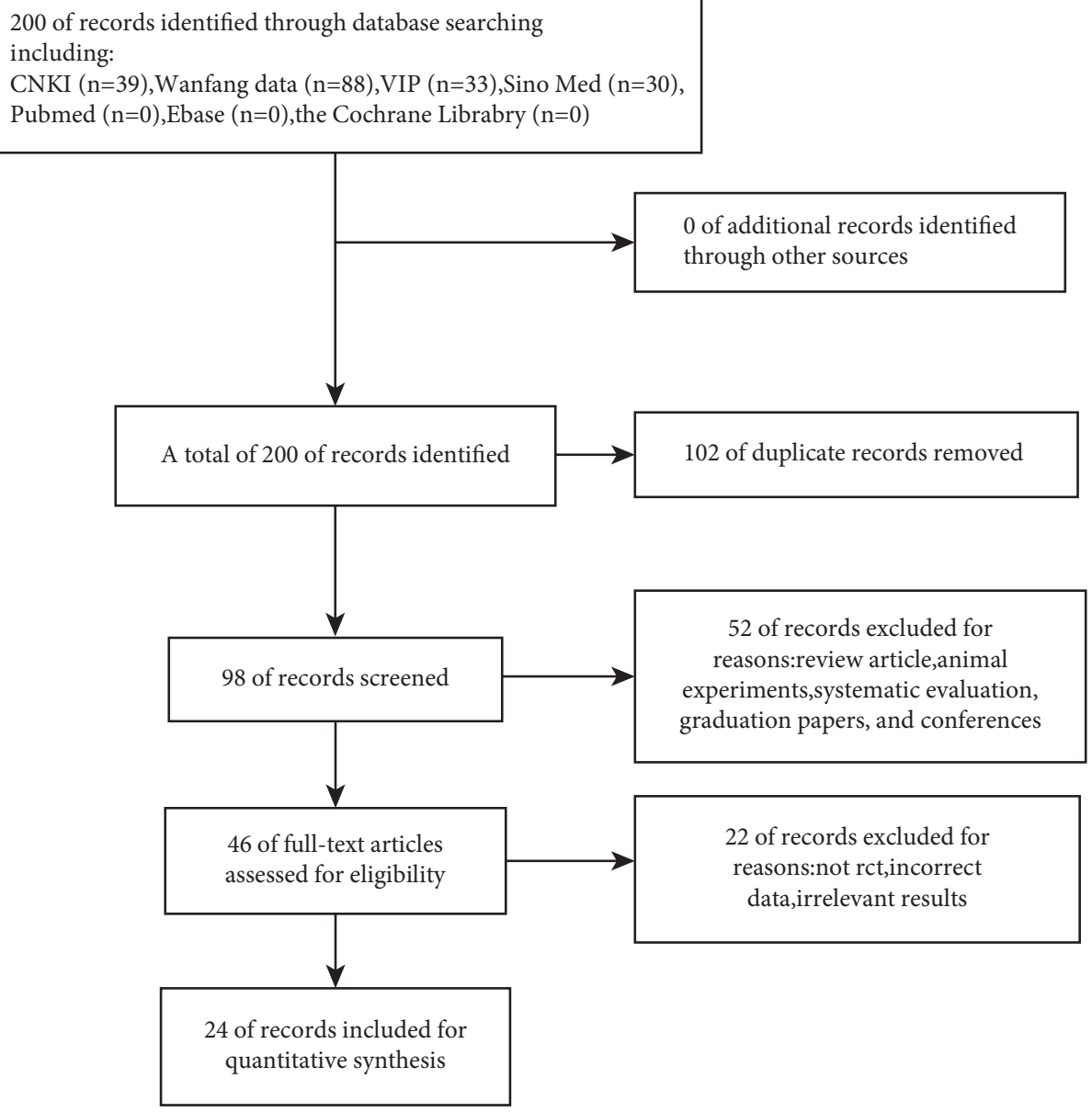

FIgURE 1: Flow diagram of search and selection process.

years. The sample size varied widely between studies (from 52 to 297 participants), and all were conducted in China and published between 2013 and 2021. Each article consisted of QJSBC treatment in the experimental group and non-QJSBC treatment in the control group. Our control group included 13 studies [21-24, 29, 30, 32-35, 39, 40, 42] using CC, 2 studies $[25,26]$ using CR, 2 studies $[27,31]$ using CC + CR, 2 studies $[22,32]$ using $\mathrm{CC}+\mathrm{ADLC}$, and 7 studies [28, 36-38, 41, 43, 44] using CT. Overall, 21 studies [21-23, 25-30, 32-38, 40-44] reported WBC, 10 studies $[21,23,27,29,30,32-34,40,42]$ reported $\mathrm{Hb}, 13$ studies $[21,23,27,29,30,32-35,40-43]$ reported PLT, 7 studies $[22,23,32,33,35,36,43]$ reported NEU, 8 studies $[24-27,31,33,35,39]$ reported BMSR, and 4 studies $[21,37,40,42]$ reported ER.

3.3. Methodological Quality. Two reviewers independently conducted a risk of bias assessment using the Cochrane Collaboration's tool. Using this method, the risk of bias of each trial was assessed based on seven components: random sequence generation (selection bias), allocation concealment (selection bias), blinding of participants and personnel (performance bias), blinding of outcome assessment (detection bias), incomplete outcome data (attrition bias), selective reporting (reporting bias), and other bias. The methodological quality assessment for each of the relevant studies is shown in Figures 2 and 3. Of these, two trials $[22,32]$ referred to blinding and allocation concealment, and three studies $[22,24,32]$ reported the withdrawal of participants from the study, which resulted in incomplete data. No selective reporting or other biases were found. Any discrepancies were resolved by discussion among all reviewers.

\subsection{Outcomes of the Indicators}

3.4.1. WBC Counts. A total of 21 research trials [21-23, 25-30, 32-38, 40-44] with 2155 patients evaluated the effect of QJSBC on WBC, with 1162 patients in the QJSBC treatment group and 993 patients as control. A meta-analysis of these results revealed that, compared to the control group, treatment with QJSBC significantly improved WBC counts $(\mathrm{MD}=1.08,95 \% \mathrm{CI}$ : $[0.90,1.26]$, $p<0.00001$; heterogeneity: $p<0.00001, I^{2}=96 \%$, random 
TABLe 1: Characteristics of the included trials.

\begin{tabular}{|c|c|c|c|c|c|c|c|c|c|}
\hline \multirow[t]{2}{*}{ Author/year } & \multicolumn{2}{|c|}{$\begin{array}{l}\text { Number } \\
\text { of } \\
\text { subjects }\end{array}$} & \multicolumn{2}{|c|}{ Male/female } & \multicolumn{2}{|c|}{ Age $:$ mean \pm SD } & \multicolumn{2}{|c|}{ Intervention } & \multirow[t]{2}{*}{$\begin{array}{c}\text { Treatment } \\
\text { duration }\end{array}$} \\
\hline & E & $\mathrm{C}$ & $\mathrm{E}$ & $\mathrm{C}$ & E & $\mathrm{C}$ & $\mathrm{E}$ & $\mathrm{C}$ & \\
\hline Chong Wang, 2020 & 38 & 38 & $22 / 16$ & $21 / 17$ & $51.00 \pm 3.50$ & $50.50 \pm 3.00$ & QJSBC + CC & $\mathrm{CC}$ & NR \\
\hline Tongde Zhao, 2020 & 192 & 95 & $77 / 115$ & $38 / 57$ & $54.70 \pm 10.66$ & $55.24 \pm 10.60$ & QJSBC + CC & $\mathrm{CC}+\mathrm{ADLC}$ & $20 \mathrm{~d}$ \\
\hline Jing Xie, 2015 & 72 & 68 & $48 / 24$ & $42 / 26$ & $60.5 \pm 4.2$ & $57 \pm 3.5$ & $\mathrm{QJSBC}+\mathrm{CC}$ & $\mathrm{CC}$ & $18 \mathrm{w}$ \\
\hline Li Zhang, 2017 & 157 & 140 & $89 / 73$ & $83 / 79$ & $37.13 \pm 12.13$ & $36.95 \pm 11.56$ & QJSBC + CC & $\mathrm{CC}$ & $12 \mathrm{w}$ \\
\hline Rongmei Ding, 2016 & 70 & 70 & $37 / 33$ & $38 / 32$ & $50-72$ & $51-73$ & QJSBC + CR & CR & $4-5 w$ \\
\hline Bo Chen, 2017 & 51 & 51 & $30 / 21$ & $33 / 18$ & $55.3 \pm 4.8$ & $56.6 \pm 4.7$ & QJSBC + CR & $\mathrm{CR}+\mathrm{CT}$ & $4 \mathrm{w}$ \\
\hline Chi Zhang, 2018 & 75 & 75 & $41 / 34$ & $43 / 32$ & $37.01 \pm 5.58$ & $36.78 \pm 5.47$ & QJSBC + CC & $\mathrm{CC}$ & $6 \mathrm{w}$ \\
\hline Fan Huang, 2020 & 68 & 68 & $36 / 32$ & $41 / 27$ & $48.41 \pm 5.91$ & $51.62 \pm 6.75$ & QJSBC + CC + CR & $\mathrm{CC}+\mathrm{CR}$ & NR \\
\hline Hong Lin, 2015 & 60 & 59 & $32 / 28$ & $29 / 30$ & $40 \pm 10$ & $41 \pm 10$ & QJSBC + CT & CT & $4 \mathrm{w}$ \\
\hline Zhenguo Song, 2018 & 50 & 50 & $28 / 22$ & $30 / 20$ & $51.3 \pm 4.2$ & $51.7 \pm 3.9$ & QJSBC + CC & CC & $8 w$ \\
\hline Yuan Gao, 2013 & 27 & 25 & $17 / 10$ & $13 / 12$ & $36-70$ & $32-70$ & QJSBC + CC & $\mathrm{CC}$ & $12 \mathrm{w}$ \\
\hline Xinhu Wu, 2013 & 30 & 60 & $17 / 13$ & $37 / 23$ & $26-75$ & $28-76$ & $\mathrm{QJSBC}+\mathrm{CC}+\mathrm{CR}+\mathrm{CT}$ & $\mathrm{CC}+\mathrm{CR}+\mathrm{CT}$ & NR \\
\hline Liyuan Lv, 2020 & 82 & 34 & $77 / 32$ & $38 / 14$ & $58.64 \pm 9.66$ & $57.77 \pm 10.37$ & QJSBC + CC + ADLC & $\mathrm{CC}+\mathrm{ADLC}$ & $20 \mathrm{~d}$ \\
\hline Shengping Li, 2019 & 32 & 32 & $18 / 14$ & $17 / 15$ & $64.34 \pm 10.03$ & $63.16 \pm 8.14$ & QJSBC + CC & $\mathrm{CC}$ & $21 \mathrm{~d}$ \\
\hline Shengtao Fan, 2018 & 30 & 28 & $15 / 15$ & $14 / 14$ & $49.5 \pm 5.6$ & $49.5 \pm 5.7$ & QJSBC + CC & $\mathrm{CC}$ & NR \\
\hline Wei Zheng, 2013 & 42 & 41 & NR & NR & $55.2 \pm 5.7$ & $55.2 \pm 5.7$ & QJSBC + CC & $\mathrm{CC}$ & $30 \mathrm{~d}$ \\
\hline Hong Yu, 2019 & 30 & 30 & $17 / 13$ & $16 / 14$ & $45.16 \pm 10.13$ & $45.33 \pm 10.29$ & QJSBC + CC & $\mathrm{CC}$ & $3 w$ \\
\hline Yi Zhang, 2013 & 46 & 40 & $28 / 18$ & $26 / 14$ & $34 \pm 14$ & $29 \pm 16$ & QJSBC + CT & $\mathrm{CT}$ & $8 w$ \\
\hline Cai Liu, 2017 & 34 & 34 & $21 / 13$ & $22 / 12$ & $38.6 \pm 1.2$ & $38.9 \pm 1.3$ & $\mathrm{QJSBC}+\mathrm{CT}$ & $\mathrm{CT}$ & $4 \mathrm{w}$ \\
\hline $\begin{array}{l}\text { Jingjing Cheng, } \\
2013\end{array}$ & 48 & 50 & $26 / 22$ & $24 / 26$ & $36.0 \pm 3.3$ & $39.0 \pm 2.7$ & QJSBC + CT & $\mathrm{CT}$ & $1 \mathrm{~m}$ \\
\hline Liyun Guan, 2015 & 50 & 50 & $64 / 36$ & $64 / 36$ & $39-69$ & $39-69$ & QJSBC + CT & CT & $28 \mathrm{~d}$ \\
\hline Hui Liu, 2016 & 43 & 42 & $22 / 21$ & $20 / 22$ & $56.3 \pm 10.2$ & $56.5 \pm 10.1$ & QJSBC + CC & $\mathrm{CC}$ & $30 \mathrm{~d}$ \\
\hline Ping Liu, 2013 & 47 & 38 & $29 / 18$ & $21 / 17$ & $14-60$ & $16-56$ & QJSBC + CT & $\mathrm{CT}$ & $4 \mathrm{w}$ \\
\hline Yuzhi Yang, 2013 & 50 & 50 & NR & NR & $21 \sim 55$ & $21 \sim 55$ & QJSBC + CT & CT & $4 \mathrm{w}$ \\
\hline
\end{tabular}

QJSBC = Qi Jiao Sheng Bai Capsule, ADLC = An Duo Lin Capsule, CC $=$ conventional chemotherapy, CT $=$ conventional treatment, CR $=$ conventional radiotherapy), $m=$ month, $\mathrm{w}=$ weeks, $\mathrm{d}=$ days, $\mathrm{NR}=$ not reported.

effects model) (Figure 4). Subsequently, a subgroup analysis was performed based on disease type, control group treatment, and treatment duration (Figures 5(a)$5(c))$. The analysis of the disease type provided the following results for the subgroups "tumor-associated leukopenia” $(\mathrm{MD}=1.17 ; 95 \%$ CI $[0.94,1.40])$, and "nontumor-associated leukopenia" (MD $=0.91$; 95\% CI $[0.52,1.29])$. The analysis based on the treatment plan of the control group provided the following results: "CC" subgroup $(\mathrm{MD}=1.08 ; 95 \%$ CI $[0.86,1.31])$; "CR" subgroup $(\mathrm{MD}=1.22 ; 95 \% \mathrm{CI}[1.05,1.38]) ;$ "CC + ADLC/ $\mathrm{CC}+\mathrm{CR}$ ” subgroup $(\mathrm{MD}=2.34 ; 95 \% \mathrm{CI}[-0.43,5.10])$; and "CT" subgroup $(\mathrm{MD}=0.84 ; 95 \% \mathrm{CI}[0.49,1.18])$. Finally, an analysis of the postmortem subgroup was performed according to the duration of treatment, showing "treatment duration less than 1 month" subgroup $(\mathrm{MD}=0.88 ; 95 \% \mathrm{CI}[0.63,1.13])$; "treatment duration longer than 1 month" subgroup (MD $=1.25 ; 95 \% \mathrm{CI}[1.03$, 1.47]); and "treatment duration unclear" subgroup $(\mathrm{MD}=1.82 ; 95 \% \mathrm{CI}[0.59,3.05])$. Based on these results, we concluded that the source of the heterogeneity of WBC counts may be related to the treatment time of the disease, whilst being independent of the disease type and treatment plan of the control group. According to the results of the funnel plot test, no publication bias was evident (Figure 6).
3.4.2. Hb Counts. The effect of QJSBC on $\mathrm{Hb}$ count was reported in 10 trials $[21,23,27,29,30,32-34,40,42]$, involving a total of 955 patients. The pooled results suggest that QJSBC might increase $\mathrm{Hb}$ counts of patients in the QJSBC treatment group $(\mathrm{MD}=18.12 ; 95 \%$ CI $[13.68,22.56]$, $p=0.00001)$, with high heterogeneity $(p<0.00001$, $I^{2}=87 \%$, random effects model) (Figure 7). A subgroup analysis was conducted according to the treatment plan of the control group with the following results: "CC" subgroup $(\mathrm{MD}=20.53 ; 95 \%$ CI $[16.27,24.80])$ and "CC+ADLC/ $\mathrm{CC}+\mathrm{CR}$ " subgroup $(\mathrm{MD}=8.82 ; 95 \%$ CI $[4.11,13.53])$ (Figure 8 ). Based on these results, we conclude that the source of heterogeneity in $\mathrm{Hb}$ counts may be related to the treatment plan of the control group. Since all the studies in this group were related to leukopenia of tumor-related diseases, subgroup analysis of disease types was not conducted. The funnel plot suggested a low probability of publication bias (Figure 9).

3.4.3. PLT Counts. PLT count was reported in 13 studies $[21,23,27,29,30,32-35,40-43]$, involving a total of 1200 patients. All studies demonstrated the therapeutic effect of QJSBC with high heterogeneity $(\mathrm{MD}=45.80,95 \% \mathrm{CI}[29.00$, 62.60], $p<0.00001$ heterogeneity: $p<0.00001, I^{2}=98 \%$, random effects model) (Figure 10). A subgroup analysis was 


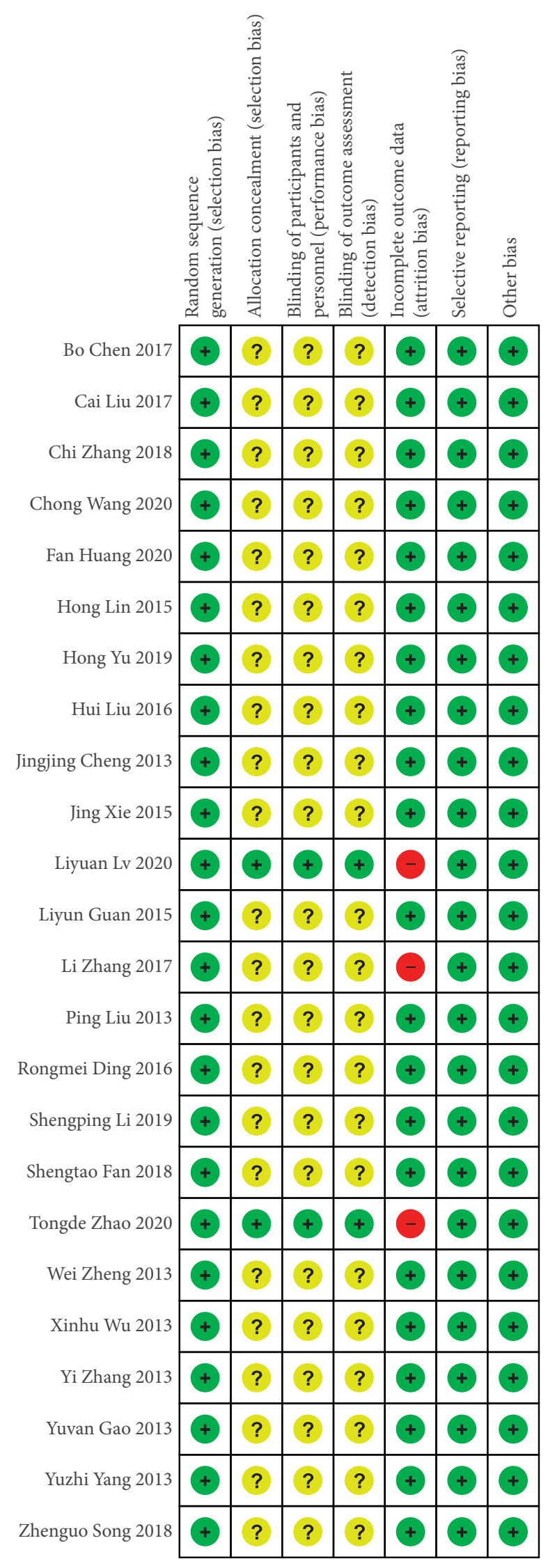

FIgURE 2: Risk of bias. performed based on disease type and the following results were obtained, as shown in Figure 11(a): "tumor-associated leukopenia" subgroup ( $\mathrm{MD}=46.30$; 95\% CI [28.81, 63.79]) and "nontumor-associated leukopenia" subgroup $(\mathrm{MD}=39.00 ; 95 \% \mathrm{CI}[12.64,65.36])$. A subgroup analysis according to the treatment plan of the control group revealed "CC" subgroup ( $\mathrm{MD}=54.32 ; 95 \%$ CI [33.69, 74.94]); “CC + ADLC/CC + CR" subgroup ( $\mathrm{MD}=-3.41$; $95 \%$ CI $[-40.08,33.26])$; and "CT" subgroup ( $\mathrm{MD}=53.72$; 95\% CI [33.12, 74.33]) (Figure 11(b)). According to these results, the source of heterogeneity of PLT counts may be related to the treatment plan of the control group and is independent of the disease type. The funnel plot was asymmetric, which suggested that there was a publication bias between the results of PLT counts (Figure 12).

3.4.4. NEU Counts. The impact of the addition of QJSBC on NEU levels was assessed in 7 studies $[22,23,32,33,35,36,43]$. The results showed that, compared with the control group, the group treated with QJSBC had significantly increased NEU counts $(\mathrm{MD}=0.59,95 \% \mathrm{CI}$ $[0.15,1.03], p=0.009$; heterogeneity: $p<0.00001, I^{2}=89 \%$, random effects model) (Figure 13). As shown in Figure 14(a), a subgroup analysis based on disease type showed "tumor-associated leukopenia" ( $\mathrm{MD}=0.44 ; 95 \% \mathrm{CI}$ $[-0.09,0.97])$ and "nontumor-associated leukopenia" subgroup $(\mathrm{MD}=0.93 ; 95 \% \mathrm{CI}[0.05,1.81])$. A subgroup analysis conducted according to the treatment plan of the control group gave the following results: "CC" subgroup $(\mathrm{MD}=0.75 ; 95 \% \mathrm{CI}[0.07,1.43])$; "CC + ADLC" subgroup $(\mathrm{MD}=-0.28 ; 95 \% \mathrm{CI}[-0.94,0.39])$; and "CT" subgroup $(\mathrm{MD}=0.93 ; 95 \%$ CI $[0.05,1.81])$ (Figure 14(b)). According to these results, the heterogeneity of the NEU counts may be related to the treatment plan of the control group and may be independent of the disease type. Because the number of RCTs included in NEU counts was less than 10, there was no funnel chart analysis for this part of data.

3.4.5. Bone Marrow Suppression Rate. BMSR were mentioned in 8 of the trials [24-27, 31, 33, 35, 39]. Based on our assessment, when compared with controls, the use of QJSBC for treatment may reduce the occurrence of bone marrow suppression $(\mathrm{RR}=0.16,95 \% \mathrm{CI}[0.12,0.22], p<0.00001$; heterogeneity: $p=0.27, I^{2}=20 \%$, fixed effects model) and there was no funnel chart analysis (Figure 15).

3.4.6. Effective Rate. The ER was mentioned in 4 studies [21, 37, 40, 42], with a total of 287 patients. There was an absence of substantial heterogeneity $(\mathrm{RR}=6.56,95 \% \mathrm{CI}$ $[2.79,15.40], p<0.0001$, heterogeneity: $p=0.95, I^{2}=0 \%$, fixed effects model) (Figure 16). The meta-analysis results revealed that compared to controls, QJSBC treatment significantly improved the ER of patients and there was no funnel chart analysis for this part of data.

3.5. Sensitivity Analysis. A sensitivity analysis was used to evaluate the robustness of the combined results. The analysis 


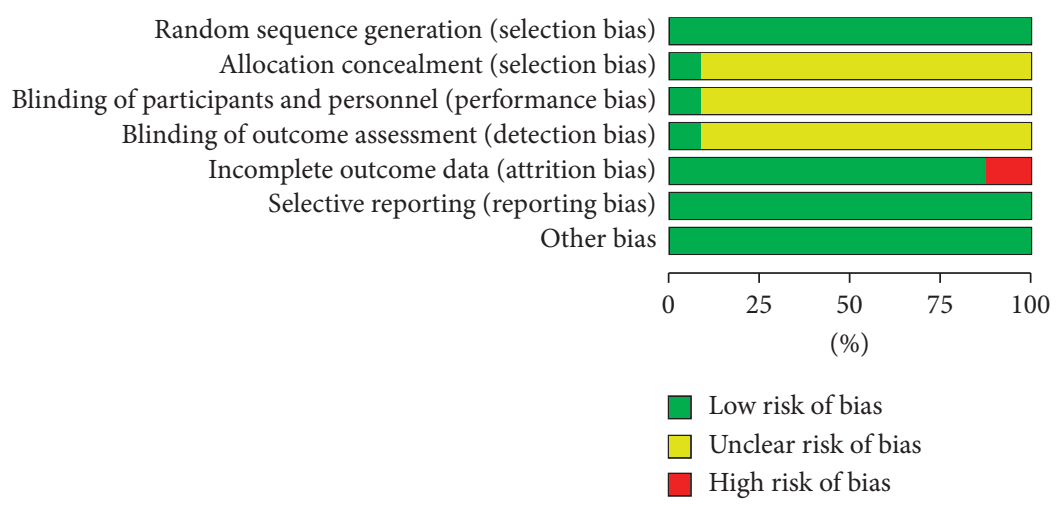

Figure 3: Risk of bias.

\begin{tabular}{|c|c|c|c|c|c|c|c|c|c|c|c|c|}
\hline \multirow{3}{*}{$\begin{array}{l}\text { Study or Subgroup } \\
\text { Bo Chen } 2017\end{array}$} & \multicolumn{3}{|c|}{ Experimental } & \multicolumn{3}{|c|}{ Control } & \multirow{3}{*}{$\begin{array}{c}\text { Weight } \\
(\%)\end{array}$} & \multirow{3}{*}{$\begin{array}{c}\begin{array}{c}\text { Mean Difference } \\
\text { IV, Random, 95\% CI }\end{array} \\
1.13[1.05,1.21]\end{array}$} & \multirow{2}{*}{\multicolumn{4}{|c|}{$\begin{array}{l}\text { Mean Difference } \\
\text { IV, Random, } 95 \% \text { CI }\end{array}$}} \\
\hline & \multirow{2}{*}{$\begin{array}{c}\text { Mean } \\
4.29\end{array}$} & \multirow{2}{*}{$\begin{array}{c}\text { SD } \\
0.25\end{array}$} & \multirow{2}{*}{$\begin{array}{c}\text { Total } \\
51\end{array}$} & \multirow{2}{*}{$\frac{\text { Mean }}{3.16}$} & \multirow{2}{*}{$\frac{\mathrm{SD}}{0.16}$} & \multirow{2}{*}{$\begin{array}{c}\text { Total } \\
51\end{array}$} & & & & & & \\
\hline & & & & & & & & & & & $=$ & \\
\hline Cai Liu 2017 & 5.17 & 0.18 & 34 & 4.18 & 0.1 & 34 & 6.2 & $0.99[0.92,1.06]$ & & & $=$ & \\
\hline Chong Wang 2020 & 5.51 & 1.33 & 38 & 4.36 & 0.96 & 38 & 4.1 & $1.15[0.63,1.67]$ & & & & \\
\hline Fan Huang 2020 & 6.63 & 2.45 & 68 & 3.44 & 1.56 & 68 & 3.3 & $3.19[2.50,3.88]$ & & & & \\
\hline Hong Lin 2015 & 4.4 & 0.4 & 60 & 4.1 & 0.4 & 59 & 6.0 & $0.30[0.16,0.44]$ & & & w & \\
\hline Hong Yu 2019 & 5.75 & 0.28 & 30 & 5.03 & 0.41 & 30 & 5.9 & $0.72[0.54,0.90]$ & & & - & \\
\hline Hui Liu 2016 & 4.26 & 0.83 & 43 & 3.73 & 0.87 & 42 & 5.0 & $0.53[0.17,0.89]$ & & & 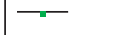 & \\
\hline Jing Xie 2015 & 4.11 & 1.09 & 72 & 2.31 & 0.98 & 68 & 5.1 & $1.80[1.46,2.14]$ & & & & \\
\hline Jingjing Cheng 2013 & 4.69 & 0.64 & 48 & 4.21 & 0.68 & 50 & 5.6 & $0.48[0.22,0.74]$ & & & - & \\
\hline Liyuan Lv 2020 & 9.3 & 4.47 & 82 & 4.88 & 3.7 & 34 & 1.1 & $4.42[2.84,6.00]$ & & & & \\
\hline Liyun Guan 2015 & 4.13 & 0.25 & 50 & 3.69 & 0.25 & 50 & 6.2 & $0.44[0.34,0.54]$ & & & $=$ & \\
\hline Ping Liu 2013 & 3.97 & 0.27 & 47 & 2.46 & 0.35 & 38 & 6.1 & $1.51[1.37,1.65]$ & & & 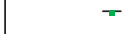 & \\
\hline Rongmei Ding 2016 & 4.3 & 0.24 & 70 & 3 & 0.18 & 70 & 6.2 & $1.30[1.23,1.37]$ & & & $=$ & \\
\hline Shengping Li 2019 & 5.78 & 1.99 & 32 & 3.82 & 2.49 & 32 & 1.9 & $1.96[0.86,3.06]$ & & & & \\
\hline Shengtao Fan 2018 & 5.52 & 1.34 & 30 & 4.35 & 0.95 & 28 & 3.8 & $1.17[0.58,1.76]$ & & & & \\
\hline Tongde Zhao 2020 & 5.51 & 2.43 & 192 & 6 & 4.64 & 95 & 2.2 & $-0.49[-1.48,0.50]$ & & & & \\
\hline Wei Zheng 2013 & 5.51 & 1.33 & 42 & 4.37 & 0.99 & 41 & 4.2 & $1.14[0.64,1.64]$ & & & $\longrightarrow$ & \\
\hline Yi Zhang 2013 & 4.5 & 1.2 & 46 & 3.3 & 1.1 & 40 & 4.3 & $1.20[0.71,1.69]$ & & & 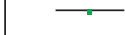 & \\
\hline Yuan Gao 2013 & 4.08 & 0.33 & 27 & 3.02 & 0.58 & 25 & 5.6 & $1.06[0.80,1.32]$ & & & - & \\
\hline Yuzhi Yang 2013 & 5.16 & 1.17 & 50 & 4.15 & 0.82 & 50 & 4.8 & $1.01[0.61,1.41]$ & & & - & \\
\hline Zhenguo Song 2018 & 4.13 & 0.22 & 50 & 3.12 & 0.24 & 50 & 6.2 & $1.01[0.92,1.10]$ & & & 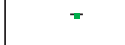 & \\
\hline Total $(95 \%$ CI) & & & 1162 & & & 993 & 100.0 & $1.08[0.90,1.26]$ & & & $\gamma$ & \\
\hline \multirow{2}{*}{\multicolumn{9}{|c|}{$\begin{array}{l}\text { Heterogeneity: } \mathrm{Tau}^{2}=0.14 ; \mathrm{Chi}^{2}=472.71, d f= \\
\text { Test for overall effect: } Z=11.61(P<0.00001)\end{array}$}} & -2 & -1 & 1 & 2 \\
\hline & & & & & & & & & Favours [exp & rimental] & Favours & s [control] \\
\hline
\end{tabular}

FIgURE 4: Forest plot for WBC.

showed that no individual study had a significant impact on the overall results. Based on the sensitivity analysis, the combined effect size of WBC counts, Hb counts, PLT counts, NEU counts, BMSR, and ER was stable.

\section{Discussion}

Leukopenia, a common blood disease with a high incidence, is also a common clinical disease symptom and reacts adversely to medication [45]. Clinically, leukopenia causes recurrent infection, especially in tumor patients, which is not conducive to further radiation and chemotherapy. Despite its rapid response, G-CSF, although commonly used, is not suitable for long-term use in all patients due to its short duration of efficacy, high cost, and the increased risk of secondary malignancy.

According to TCM theory, leukopenia belongs to the category of "deficiency of fatigue" (Xu lao) [46], with most patients having a deficiency of both Qi and blood. Thus, QJSBC is used for treatment as it has the effect of enriching blood and replenishing Qi [47]. TCM doctors tend to use proprietary Chinese medicines flexibly based on TCM theories, which often results in a lack of adequate evidencebased support for the treatment of diseases with proprietary Chinese medicines.

According to the current results from randomized controlled trials, QJSBC combined with western medicine can help to increase the count of WBC, Hb, PLT, and NEU; reduce the BMSR; and improve the ER. Many of the published studies [24, 31, 39] did not include all of these indicators, all of which show the advantage of QJSBC in improving the BMSR. In addition, the proprietary Chinese medicine QJSBC is generally safe, with no studies finding that it caused serious adverse events. A subgroup analysis of outcome indicators with heterogeneity was conducted to find the source of the heterogeneity. We 


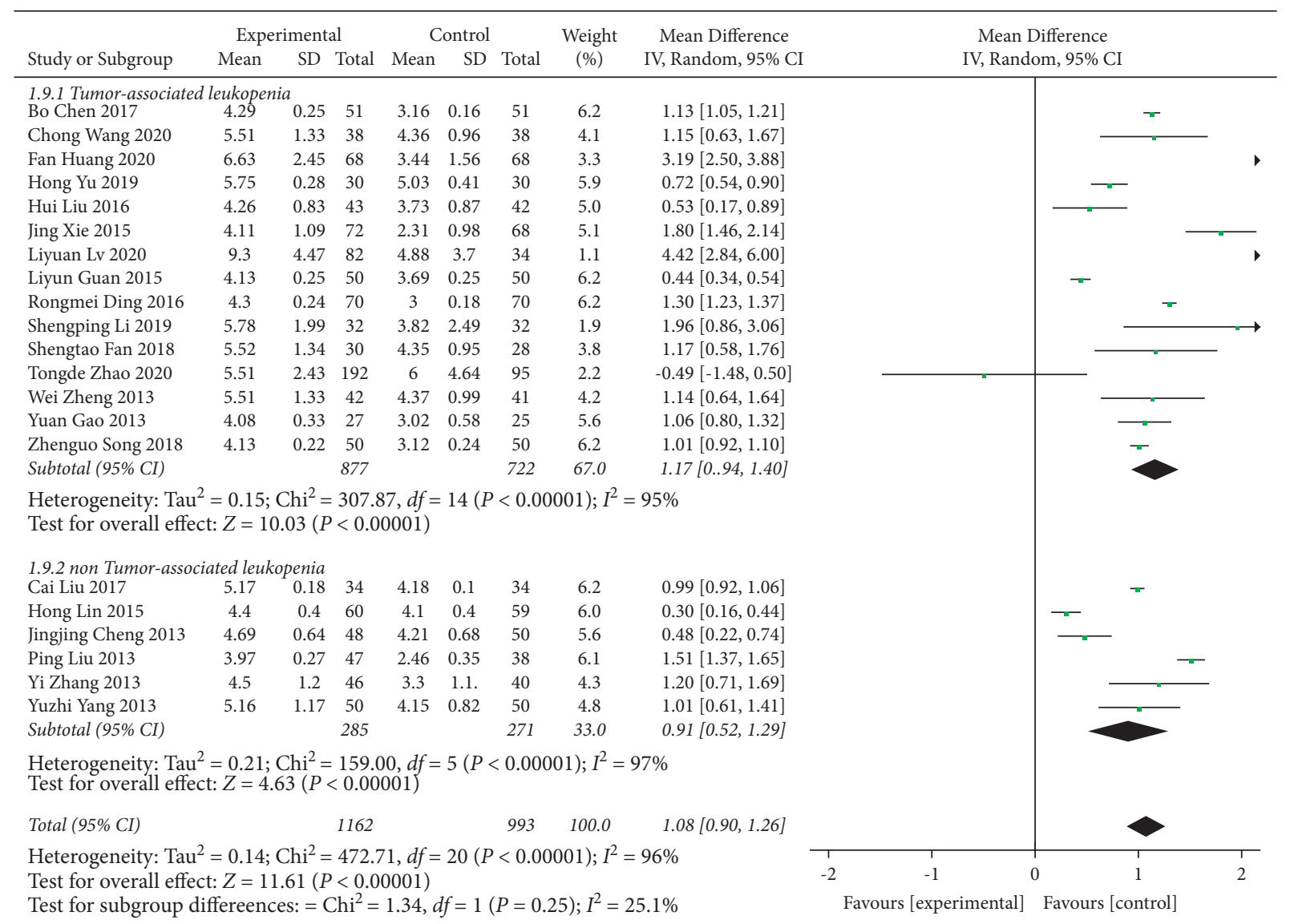

(a)

FIGURE 5: Continued. 


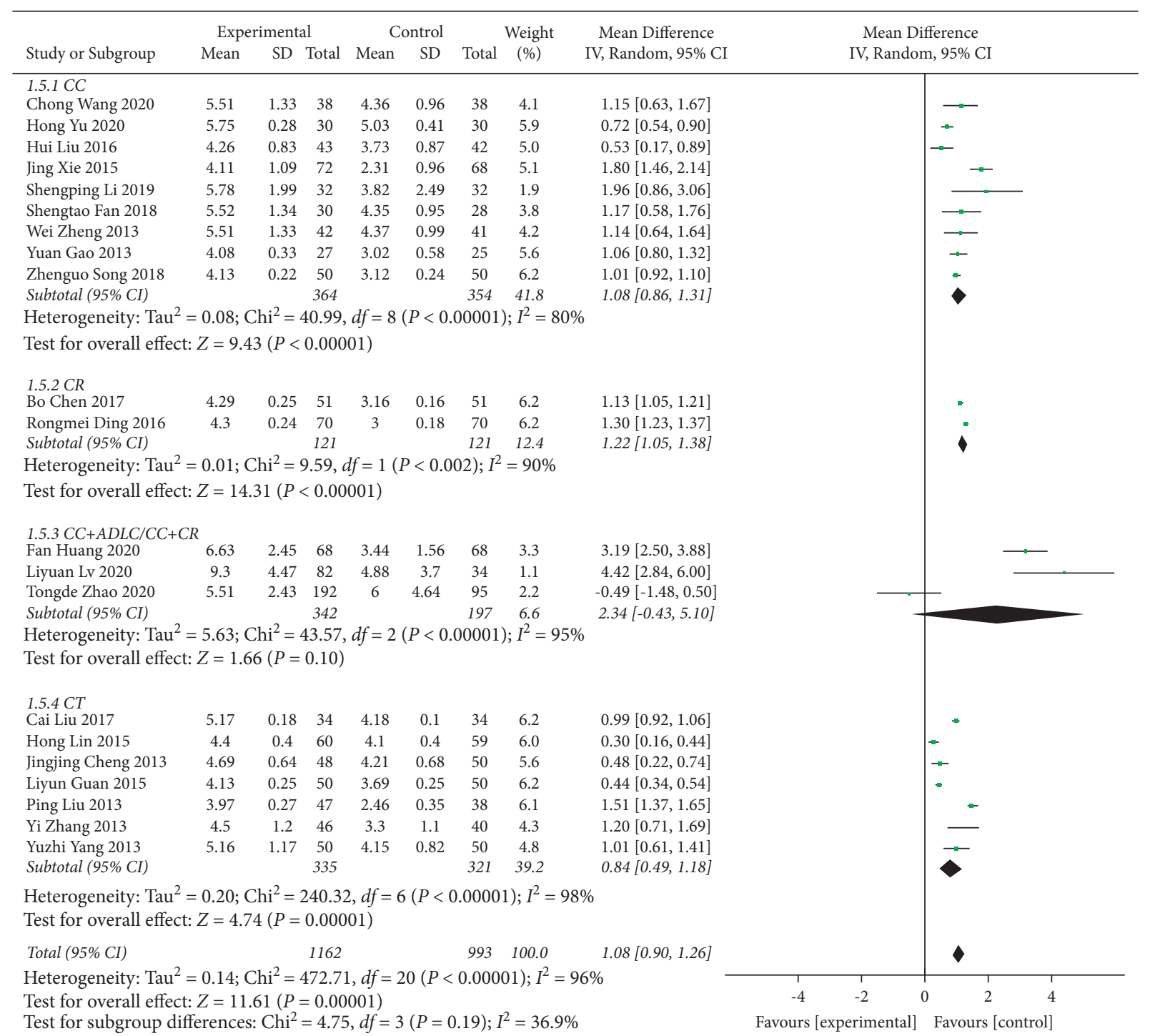

(b)

FIgUre 5: Continued. 


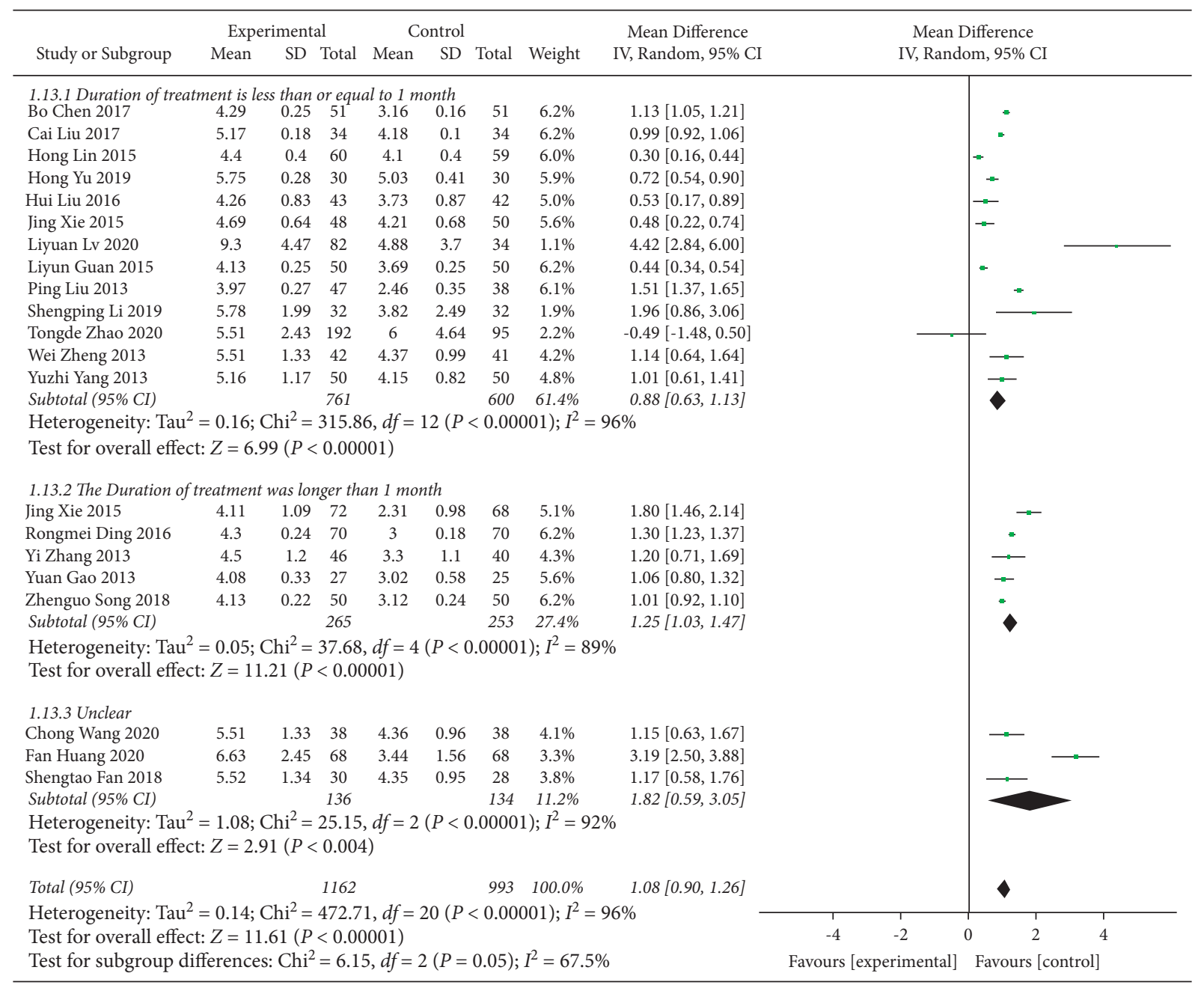

(c)

FIGURE 5: Forest plot of WBC subgroup analysis: (a) disease type, (b) control group treatment, (c) treatment duration.

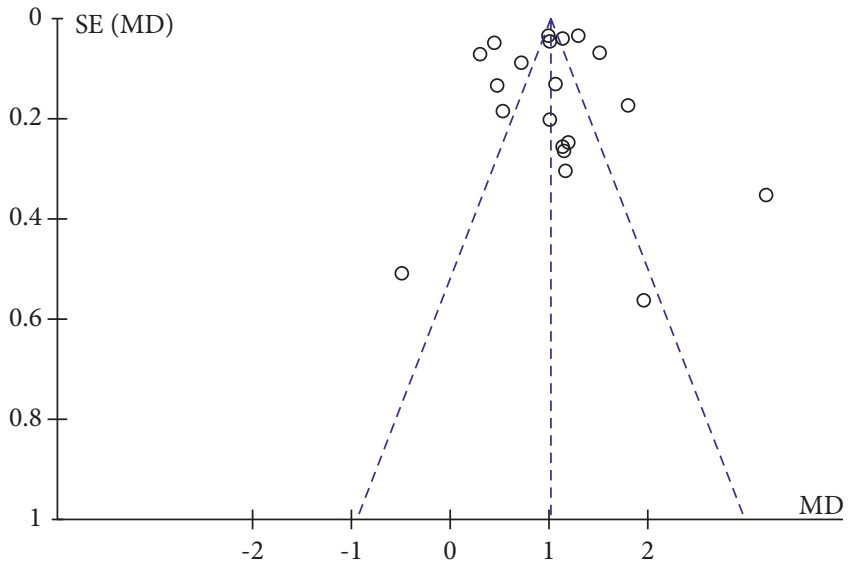

FIgURE 6: Funnel plot for the publication bias of WBC.

found that the heterogeneity may be related to the type of disease, the treatment plan, and the duration of treatment. The funnel plot of PLT counts suggested that there was a publication bias. Sensitivity analysis showed that the results for WBC, Hb, PLT, NEU, BMSR, and ER were robust for all changes.

A meta-analysis [4] published in 2015 examined the efficacy of QJSBC. However, flaws exist in this article. First, the authors searched a small number of databases and second, the authors used inappropriate methods when sorting the data and interpreting the results. We consider the results of the 2015 article misleading and suggest that the methods used in our study are more appropriate and effective, giving more reliable results.

Systematic reviews and meta-analyses are at the top of the hierarchy of clinical evidence, and we have used this approach to determine the efficacy of QJSBC on patients with leukopenia, aiming to provide an up-to-date clinical evaluation of treatment with QJSBC. This effective and lowcost Chinese patented medicine has the potential to benefit an increasing number of leukopenia patients around the world, helping to somewhat reduce the economic burden on patients, their families, and society. 


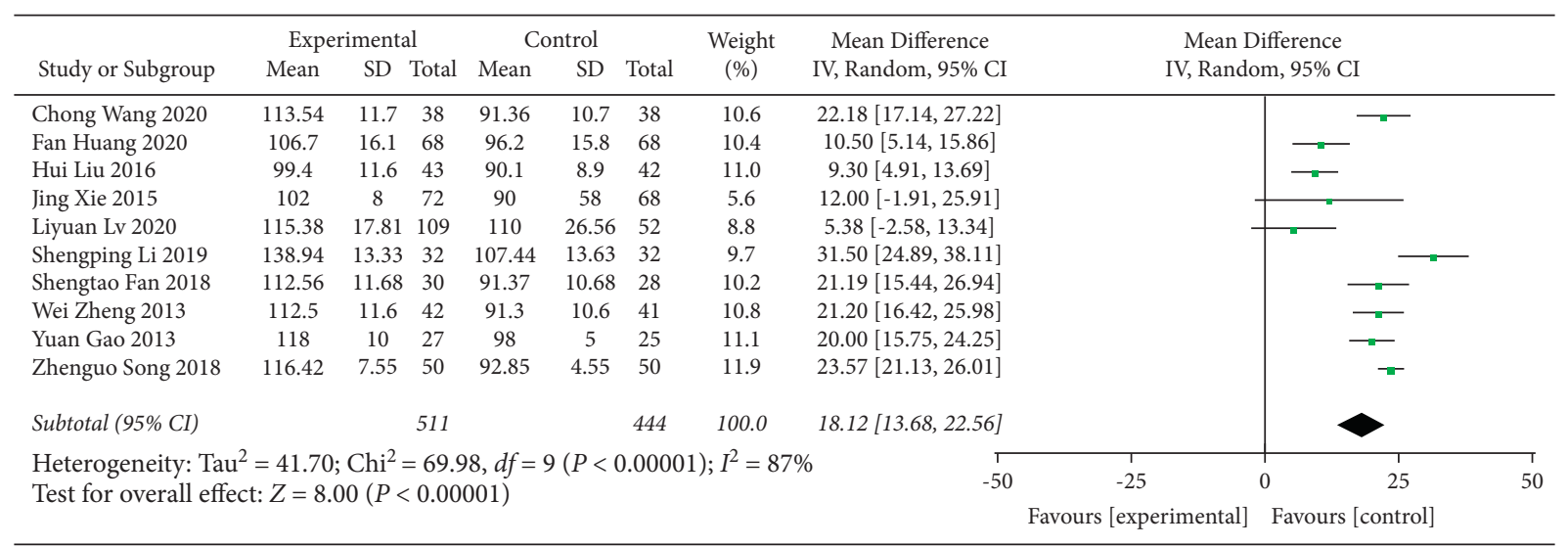

Figure 7: Forest plot of $\mathrm{Hb}$.

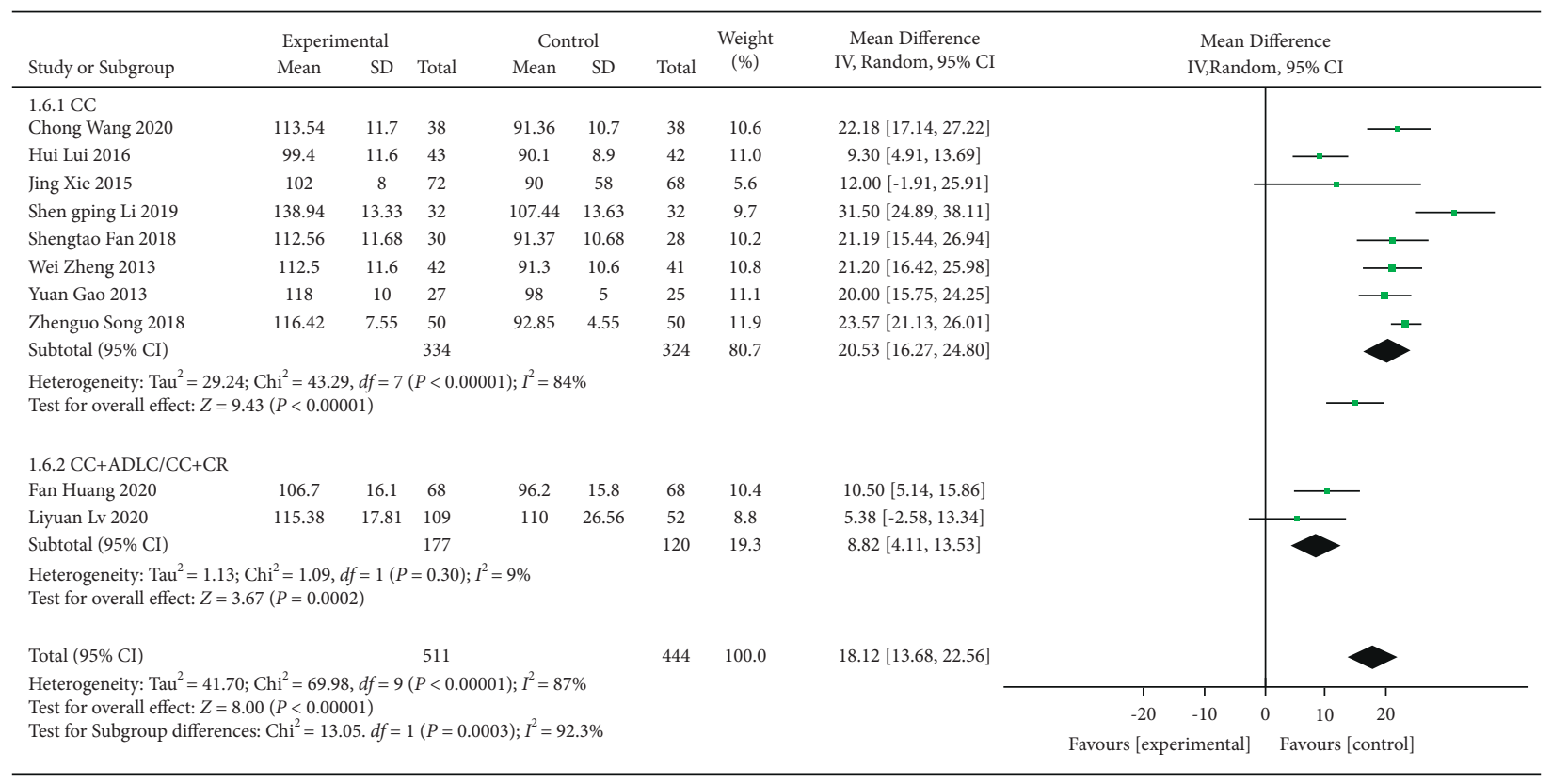

FIgURE 8: Forest plot of $\mathrm{Hb}$ subgroup analysis.

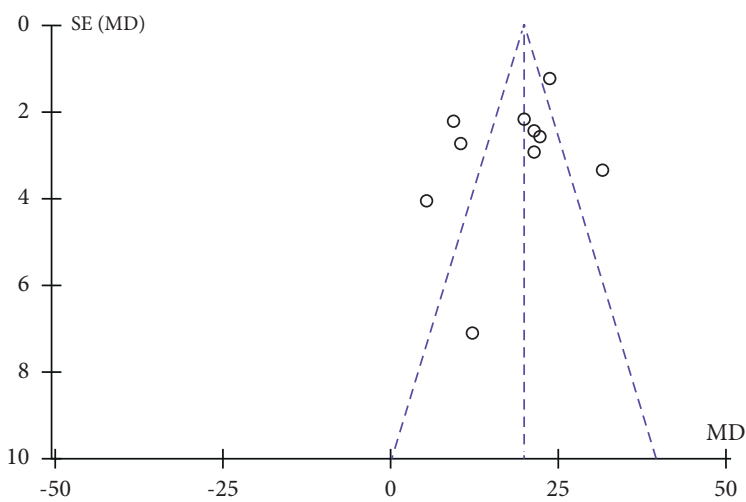

Figure 9: Funnel plot for the publication bias of $\mathrm{Hb}$. 


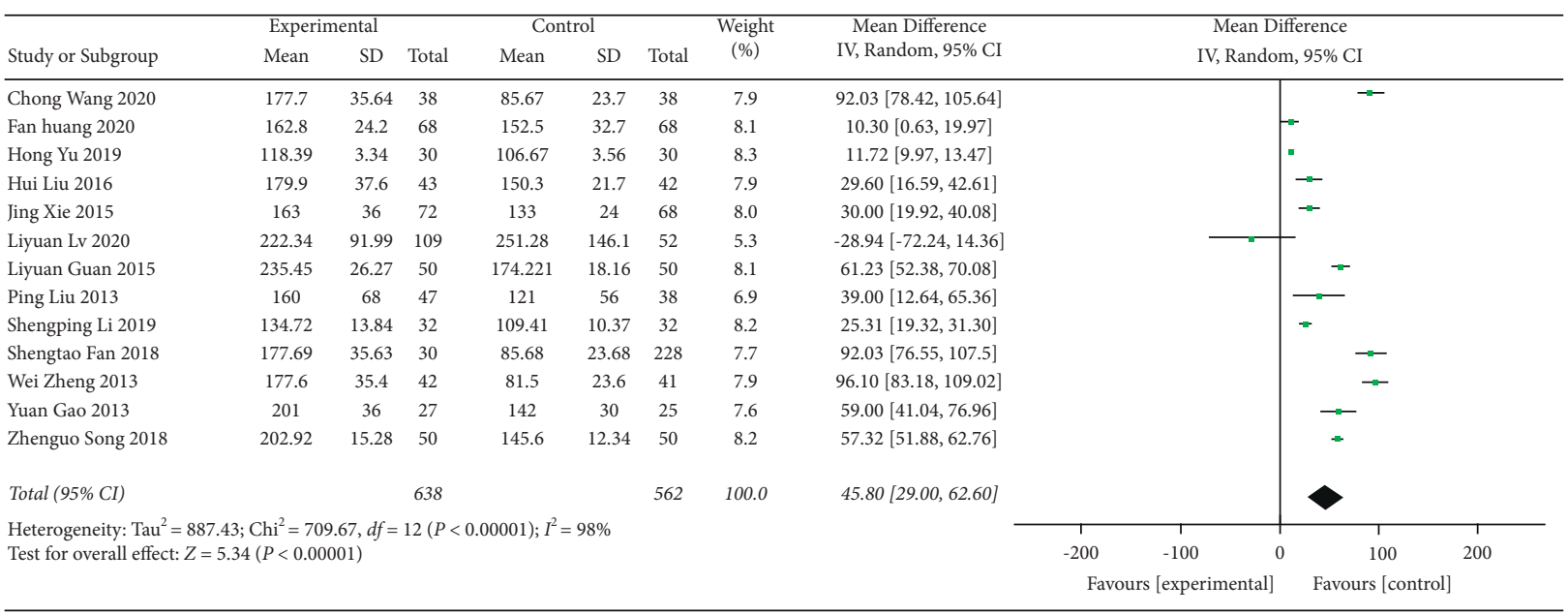

Figure 10: Forest plot of PLT.

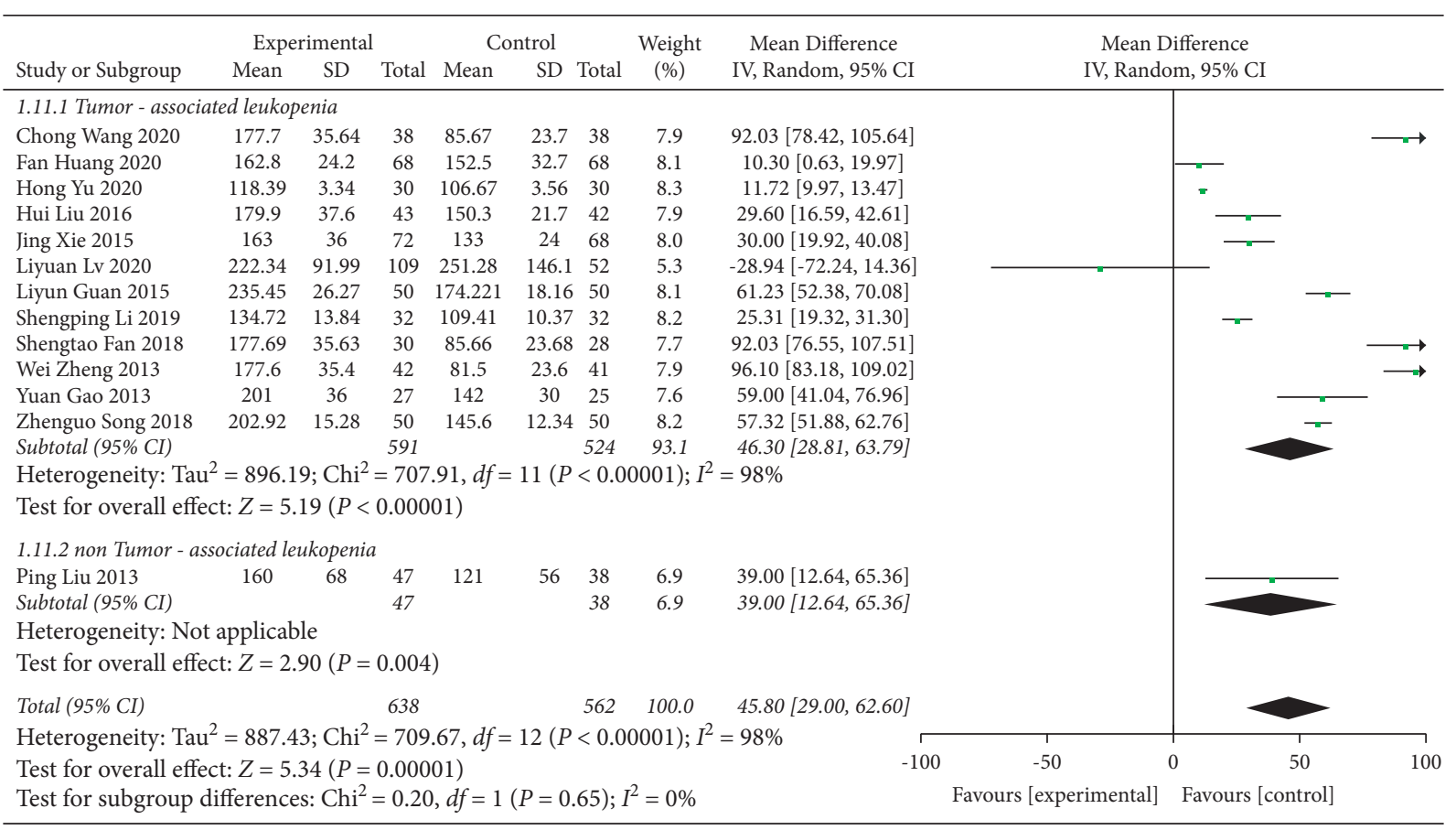

(a)

Figure 11: Continued. 


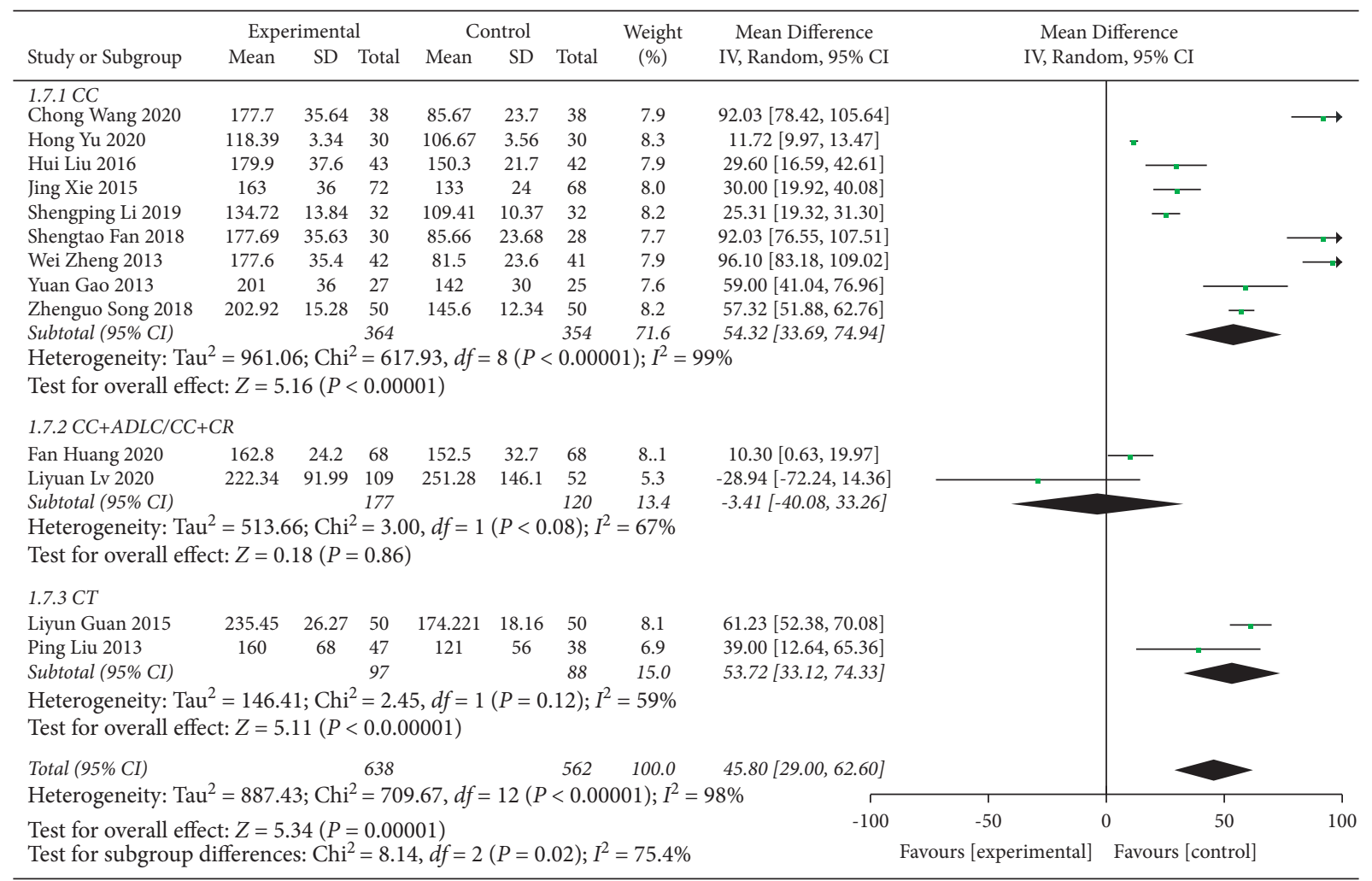

(b)

Figure 11: Forest plot of PLT subgroup analysis: (a) disease type and (b) control group treatment.

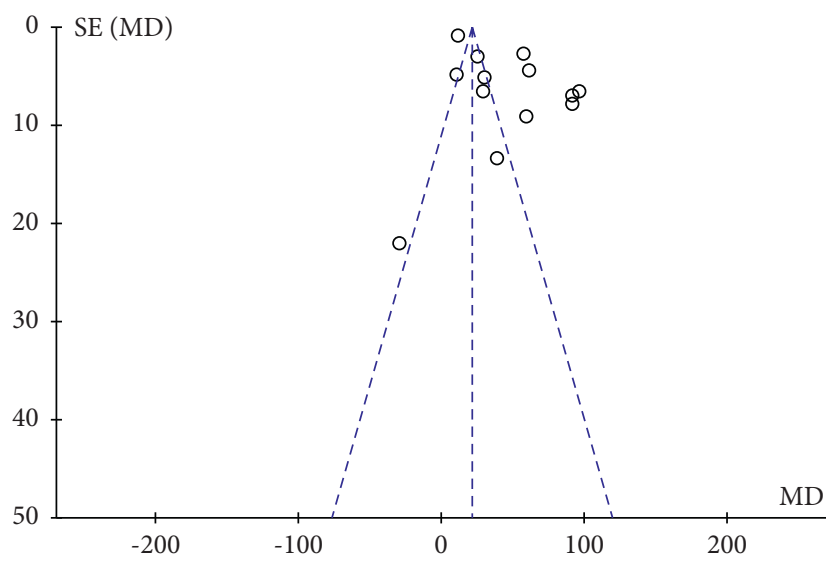

FIGURE 12: Funnel plot for the publication bias of PLT.

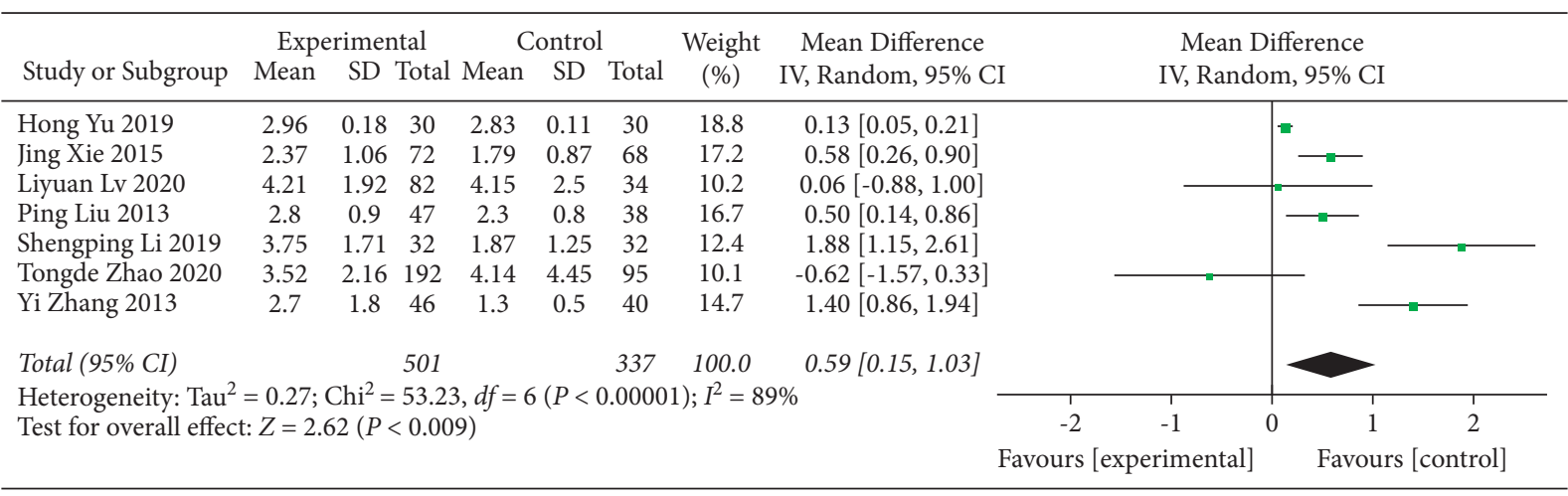




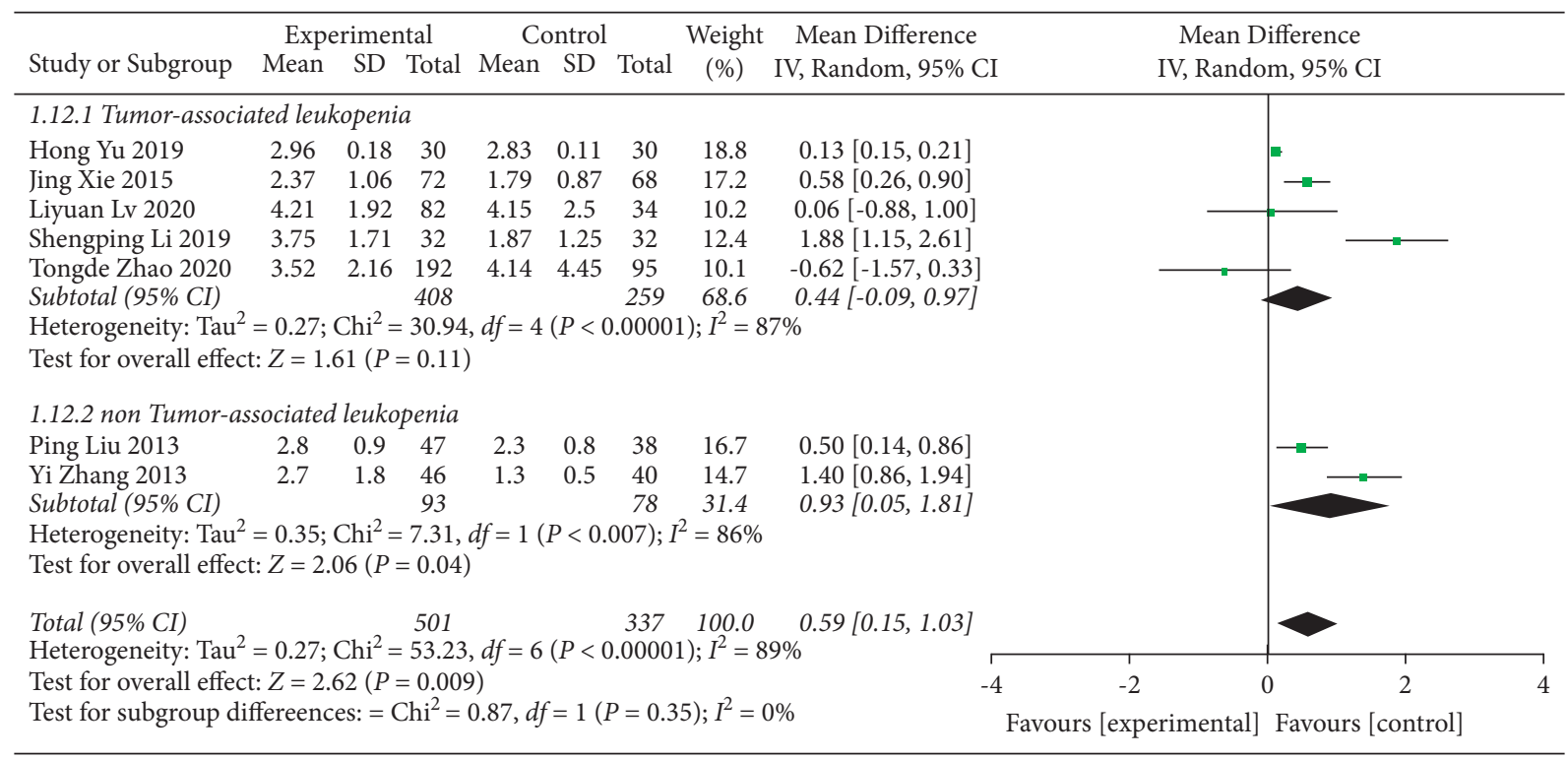

(a)

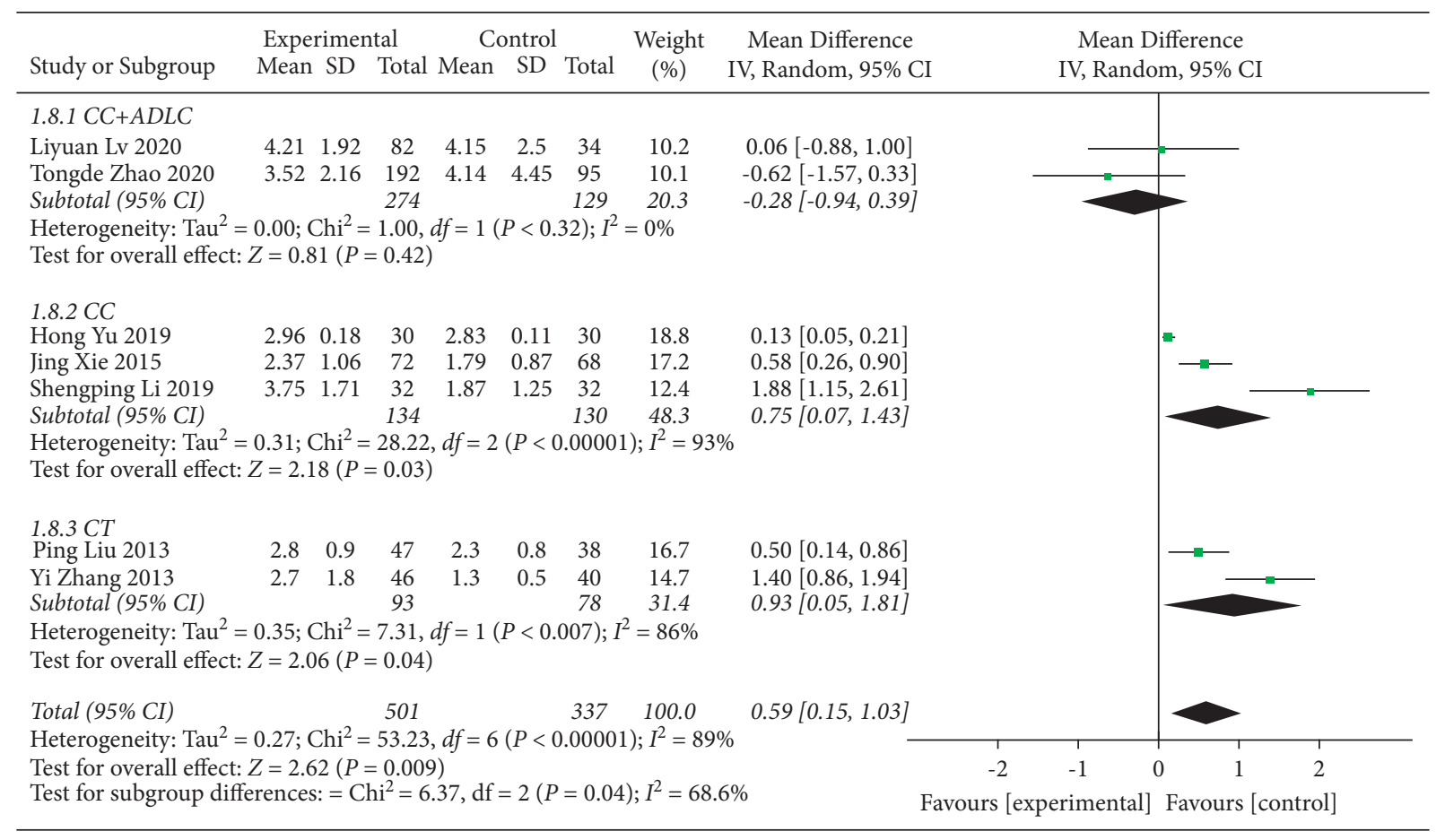

(b)

FIgURE 14: Forest plot of NEU subgroup analysis: (a) disease type and (b) control group treatment.

\section{Limitations}

Throughout the study, we have taken care of interpreting our results carefully; however, there are some limitations to consider. (1) All the included studies were published in Chinese and all the patients were Chinese, so it is not clear whether the results apply to patients from other regions. (2) There were only two high-quality, multicenter, randomized, double-blind studies. The inclusion of other studies that not meeting these criteria resulted in a lack of convincing results. The overall methods included in these studies were of low quality due to incomplete data collection, and deficiencies in 


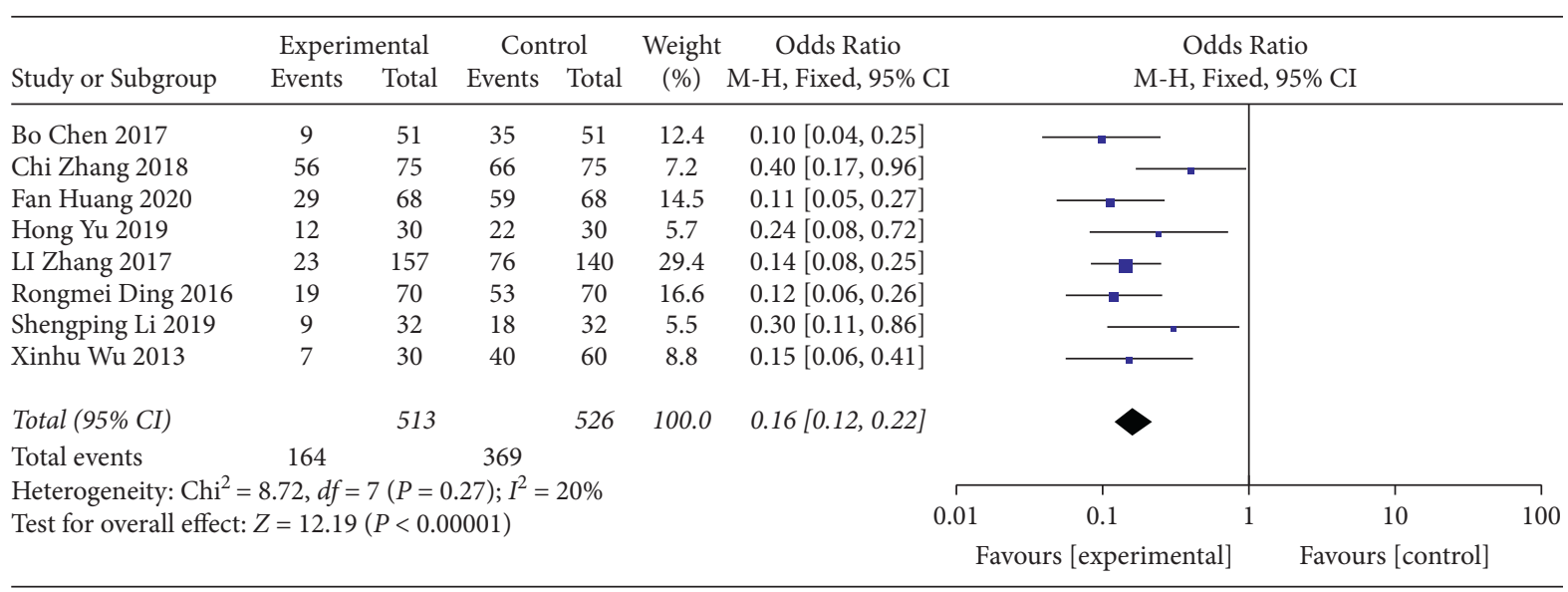

Figure 15: Forest plot of BMSR.

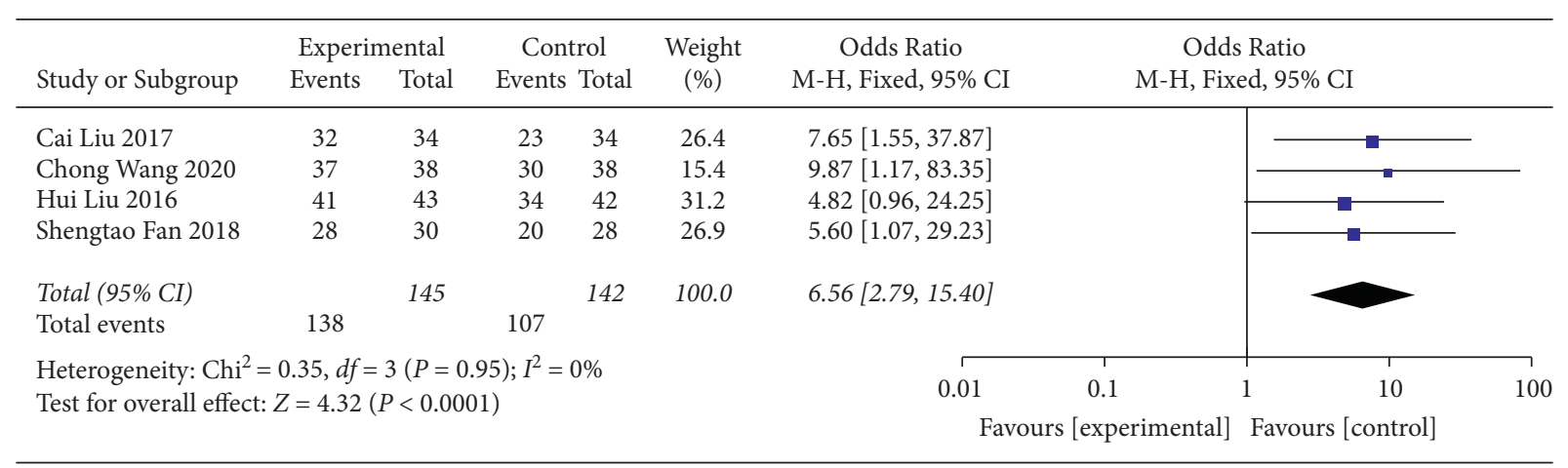

FIgURe 16: Forest plot of ER.

study design. More large-sample, multicenter, high-quality randomized controlled trials are needed for validation. (3) The fact that only 1 out of 24 trials was publicly registered means we are not able to rule out the possibility of publication bias.

\section{Conclusion}

In conclusion, this systematic review and meta-analysis evaluated the effect of QJSBC for treating leukopenia in cancer patients and comprehensively analyzed the results of randomized controlled trials published between 2013 and 2021. The systematic review and meta-analysis showed that QJSBC when compared with a non-QJSBC control group had more beneficial effects on several clinical indicators (WBC, Hb, PLT, NEU, BMSR, and ER). Although QJSBC was effective in treating leucopenia in all 24 studies, larger sample sizes and higher-quality randomized controlled trials are needed to reduce study heterogeneity and validate these findings.

\section{Conflicts of Interest}

The authors declare that they have no conflicts of interest.

\section{Authors' Contributions}

All the authors participated in the study design, data collection, statistical analysis, result explanation, manuscript drafting, and proofreading. All the authors agreed to the final version of the manuscript to be submitted. Study selection and data extraction were conducted by Hongfang $\mathrm{Fu}$ and Yilan Wang. Yang Zhang and Haoyue Feng performed the data analysis. The article was written by Hongfang Fu and revised by Yilan Wang and Xiaoyu Hu.

\section{References}

[1] L. Liu, W. C. Dan, and Q. Y. He, "Study on the mechanism of leucopenia induced by Sanguelba shengbai tablet based on network pharmacology," Modern Chinese Traditional Medicine, vol. 22, no. 9, pp. 1494-1501, 2020.

[2] J. Li, T. L. Y. Tuo, and C. Zhang, "Clinical efficacy and mechanism of bazhen decoction on leukocyte reduction induced by chemotherapy in lung cancer," World Chinese Medecine, vol. 15, no. 1, pp. 94-98, 2020.

[3] Y. Qiu and C. Y. Li, "Risk factors and prevention counter measures for leukopenia complicated with infection in cancer patients with chemotherapy," Journal of Clinical Medicine in Practice, vol. 20, no. 9, pp. 57-59, 2016. 
[4] W. Tang, J. L. Tan, and L. L. Jia, "Efficacy of qijiao shengbai capsule in the adjuvant treatment of leucopenia:A metaanalysis," China Pharmacy, vol. 26, no. 33, pp. 4672-4674, 2015.

[5] J. X. Liu and J. S. Li, "Experience of TCM treating drug-induced leukopenia with tongbu instead of bu," Journal of Ningxia Medical University, vol. 34, no. 4, pp. 415-417, 2012.

[6] C. X. Dong and M. F. Wang, "Observation of clinical effect of caffeic acid tablet on leukopenia," Chinese Remedies and Clinics, vol. 12, pp. 1714-1715, 2014.

[7] W. Xiang, Y. T. Xie, and H. Zhang, "Clinical efficacy of Yiqi Shengbai Decoction in treating leukopenia after chemotherapy of non-small cell lung cancer," Journal of Modern Oncology, vol. 27, no. 12, pp. 2117-2121, 2019.

[8] L. M. Cao, J. H. Liu, and S. L. Huang, "Nursing of granulocytosis after chemotherapy in cancer patients," Journal of Mudanjiang Medical College, vol. 28, no. 4, p. 104, 2007.

[9] Y. Zhu, D. H. Guo, and C. Yao, "Analysis of 2808 cases of spontaneous report associated with drug-induced leukopenia," The Chinese Journal of Clinical Pharmacology, vol. 36, no. 12, pp. 1719-1722, 2020.

[10] T. Y. Quan, W. Y. Chen, and G. H. Huang, "Clinical efficacy and safety evaluation of cornu cervi degelatinatum in the treatment of leukopenia after chemoradiotherapy of malignant tumor," The Chinese Journal of Clinical Pharmacology, vol. 31, no. 19, pp. 1934-1936, 2013.

[11] C. Chen, Q. W. Ding, and Z. L. Chen, "An overview of Chinese medicine treatment for leukopenia after chemotherapy for malignant tumors," Journal of New Chinese Medicine, vol. 50, no. 10, pp. 18-21, 2018.

[12] L. F. Zhao, H. J. Ma, and K. X. Zeng, "Clinical observation of acupuncture to improve leukopenia after tumor radiotherapy and chemotherapy," Liaoning Journal of Traditional Chinese Medicine, vol. 46, no. 7, pp. 1521-1522, 2019.

[13] Y. Y. Liu, J. W. Zhang, and B. Wang, "Research on the literature of medicated diet for leukopenia of radiotherapy and chemotherapy in recent 30 years," Heilongjiang Journal of Traditional Chinese Medicine, vol. 45, no. 5, pp. 69-70, 2016.

[14] Y. J. Ji and X. H. Xue, "Research status of moxibustion in the prevention and treatment of chemotherapeutic myelosuppression of malignant tumors," Guangming Journal of Chinese Medicine, vol. 34, no. 14, pp. 2266-2271, 2019.

[15] L. Yan, J. L. Liu, and Y. C. Wu, "Research progress in treatment of leukopenia after chemotherapy by traditional Chinese medicine external therapy," Chinese Journal of Acupuncture and Moxibustion (Electronic Edition), vol. 9, no. 2, pp. 72-74, 2020.

[16] L. S. Chen, T. J. Chen, and S. D. Fei, "Effect of qijiaoshengbai capsule on bone marrow depression in patients with head and neck cancer after radiotherapy," Medical and Pharmaceutical Journal of Chinese People's Liberation Army, vol. 29, no. 7, pp. 33-36, 2017.

[17] X. J. Hou, W. Li, and J. F. Zhang, "Progress in pharmacological and clinical research of qijiaoshengbai capsule," Studies of Trace Elements and Health, vol. 35, no. 1, pp. 74-76, 2018.

[18] D. Moher, A. Liberati, J. Tetzlaff, D. G. Altman, and P. Group, "Preferred reporting items for systematic reviews and metaanalyses: the PRISMA statement," BMJ, vol. 339, Article ID b2535, 2009.

[19] J. C. Zhou, Practical Oncology Internal Medicine, Vol. 23, People's Medical Publishing House, Beijing, China, 1998.

[20] J. P. Higgins, D. G. Altman, P. C. Gøtzsche et al., "The Cochrane Collaboration's tool for assessing risk of bias in randomised trials," BMJ, vol. 343, Article ID d5928, 2011.
[21] C. Wang, "Study on the effect of Qijiaoshengbai capsule combined with chemotherapy on leukopenia of tumor patients," Special Health, vol. 14, p. 79, 2020.

[22] R. M. Ding, P. Wang, and L. J. Ma, "Curative effect of Qijiao Shengbai capsule in patients with lung cancer radiotherapy leukopenia and the quality of life and influence," Journal of Modern Oncology, vol. 24, no. 3, pp. 400-403, 2016.

[23] J. Xie, Q. Liu, and L. Z. Wu, "Effect of qijiaoshenbai capsule on bone marrow suppression and immune state of ma-lignant gastrointestinal tumor after chemotherapy," The Practical Journal of Cancer, vol. 10, pp. 1462-1465, 2015.

[24] L. Zhang, Z. X. Bao, and C. W. Feng, "Effect of qijiao shengbai capsuleon bone marrow suppression of patients with NHL," Chinese Archives of Traditional Chinese Medicine, vol. 35, no. 6, pp. 1533-1535, 2017.

[25] T. D. Zhao, S. D. Tian, and L. Hou, "Clinical study of miao nationality medicine QIjiao shengbai capsule on prevention and treatment of chemotherapy related leukopenia," World Journal of Integrated Traditional and Western Medicine, vol. 15, no. 8, pp. 1547-1552, 2020.

[26] B. Chen and W. J. Wu, "Effect of Qijiao Shengbai Capsules on leukopenia in lung cancer patients after radiotherapy and its influence on quality of life," China Modern Medicine, vol. 24, no. 32, pp. 53-55, 2017.

[27] F. Huang, X. Zou, and J. Lin, "Effect of Qijiao Shengbai capsule on bone marrow suppression after preoperative chemoradiotherapy for resectable middle and low rectal cancer," Strait Pharmaceutical Journal, vol. 32, no. 12, pp. 209-210, 2020.

[28] H. Lin and H. Liao, "Observation of the curative effect of Astragalus glue rise white capsule to reduce white cells induced by clozapine," China Journal of Pharmaceutical Economics, no. 2, pp. 35-37, 2015.

[29] Z. G. Song, Z. He, and Y. L. Su, "Effects of Qijiao Shengbai capsule on immune status and quality life of patients with esophageal cancerchemotherapy," Shaanxi Journal of Traditional Chinese Medicine, vol. 39, no. 6, pp. 743-745, 2018.

[30] Y. Guan, Y. L. Wang, and J. Feng, "Effect of Qijiao Shengbai Capsule on bone marrow suppression and immune function in patients with advanced gastric cancer undergoing chemotherapy," Journal of Practical Oncology, vol. 28, no. 6, pp. 657-659, 2013.

[31] L. Wu, L. Jiang, and Y. Deng, "Preventive effect of Qijiao Shengbai Capsule on myelosuppression in patients with nasopharyngeal cancer during concurrent," Journal of Practical Oncology, vol. 28, no. 2, pp. 203-206, 2013.

[32] L. Y. Lv, P. Lv, and Y. Duan, "Clinical study on prevention and treatment of leukopenia (qi and blood deficiency syndrome) Caused by chemotherapy of lung cance with QijiaoShengbai capsule," World Chinese Medecine, vol. 15, no. 1, pp. 1-12, 2020.

[33] S. P. Li and J. H. Zhou, "Clinical observation on the treatment of bone marrow suppression after chemotherapy of non-small cell lung cancer with Qigaoshengbai capsule combined with rhG-CSF," Clinical Journal of Chinese Medicine, vol. 31, no. 7, pp. 1352-1355, 2019.

[34] W. Zheng, L. L. Zhou, and Y. Yang, "Therapeutic efficacy and bone marrow protection of Qijiao Shengbai Capsule for cancer patients with chemotherapy," Journal of Practical Oncology, vol. 28, no. 4, pp. 425-428, 2013.

[35] H. Yu, W. T. Wang, and Z. B. Chen, "Observation on the curative effect of qidaoshengbai capsule combined with granulocyte colony stimulating factor on bone marrow suppression after cancer chemotherapy," Modern Journal of 
Integrated Traditional Chinese and Western Medicine, vol. 28, no. 6, pp. 621-623, 2019.

[36] Y. Zhang, Q. Zhang, and D. D. Zhang, "Clinical study of Qi Jiao Sheng Bai capsule preventing and improving myelosuppression caused by PEG-IFN," Practical Pharmacy and Clinical Remedies, vol. 16, no. 2, pp. 102-104, 2013.

[37] C. Liu, Z. F. Li, and H. Q. Zhu, "Analysis of curative effect of qidaoshengbai capsule on leukopenia," Journal of Clinical Medical Literature (Electronic Edition), vol. 4, no. 42, p. 8258, 2017.

[38] J. J. Cheng, "Clinical observation of Qizhao Shengbai Capsule in the treatment of leucopenia caused by paliperidone," Zhejiang Journal of Integrated Traditional and Western Medicine, vol. 23, no. 5, p. 403, 2013.

[39] C. Zhang, J. R. Zhang, and W. J. Tong, "Study and analysis on clinical drug efficacy of Qijiaoshengbai capsule on the treatment of non-small cell lung cancer patients with neutropenia caused by chemotherapy," Military Medical Journal of Southeast China, vol. 20, no. 1, pp. 26-29, 2019.

[40] S. .T. Fan and Y. Ni, "Study on the effect of qidaoshengbai capsule combined with chemotherapy on leukopenia in tumor patients," Shenzhen Journal of Integrated Traditional Chinese and Western Medicine, vol. 28, no. 21, pp. 28-29, 2018.

[41] L. Y. Guan, J. R. Qu, and Q. S. Wang, "Qigaoshengbai capsule to prevent and treat primary liver cancer 50 cases of bone marrow suppression after interventional operation," China Pharmaceuticals, vol. 18, p. 108, 2015.

[42] H. Liu, "Clinical study of Qizhijiao Shengbai Capsule on leukopenia in patients with tumor chemotherapy," Asia- $\mathrm{Pa}$ cific Traditional Medicine, vol. 12, no. 22, pp. 119-120, 2016.

[43] P. Liu, "Clinical observation of qidaoshengbai capsule in the treatment of myelosuppression induced by interferon," Guide of China Medicine, vol. 32, pp. 209-210, 2013.

[44] Y. Z. Yang, "Observation of curative effect of qidaoshengbai capsule on leukopenia," Guide of China Medicine, vol. 41, no. 6, pp. 31-32, 2013.

[45] Y. T. Lin, X. Y. Zheng, and Y. F. Yao, “Therapeutic effect of spleen low molecular weight extracts on leukopenia caused by epirubicin in mice and its mechanism," Journal of Experimental Hematology, vol. 29, no. 3, pp. 969-974, 2021.

[46] J. Li, M. S. Wang, and H. Fan, "Experience summary of diagnosis and treatment of TCM in leukopenia," Tianjin Journal of Traditional Chinese Medicine, vol. 30, no. 12, pp. 732-734, 2013.

[47] H. Li, J. Chen, and C. H. Xu, "Observation on the effect of qiguaishengbai capsule on prevention and treatment of myelosuppression induced by radiotherapy for cervical cancer," Zhejiang Medical Journal, vol. 21, pp. 1942-1943, 2013. 